\title{
Spatio-temporal cross-city comparison using multi- sensoral remote sensing for Mexican cities
}

\author{
H. Taubenböck ${ }^{1}$, M. Klotz ${ }^{1}$, A. Felbier ${ }^{1}$, M. Wegmann ${ }^{2} \&$ R. Ludwig ${ }^{3}$ \\ ${ }^{1}$ German Remote Sensing Data Center (DFD). German Aerospace Center (DLR). 82234 Wessling-Oberpfaffenhofen. Germany \\ ${ }^{2}$ University of Würzburg. Institute of Geography. Am Hubland. D-97074 Würzburg \\ ${ }^{3}$ Ludwig-Maximilians University, Department of Geography, München
}

\begin{abstract}
Our planet is more and more transforming into an urban world, in which the dynamics of urbanization have overcome the ability to govern cities. In a situation of uncoordinated urban growth, regional and urban planning lack technologies and methodologies to measure, monitor and analyze the spatio-temporal pattern of dynamic urban sprawl. This paper focuses on methods using remote sensing data to analyze, quantify and compare spatial urbanization processes. Urban sprawl is detected at the level of urban footprints using a postclassification change detection approach based on multi-sensoral Landsat and TerraSAR-X data. Spatio-temporal analysis combines absolute parameters (e.g. areal growth), location-based zonal statistics and gradient analysis (e. g. urban core versus the urban fringes) as well as spatial metrics (e.g. Largest Patch Index) to quantitatively characterise the spatial pattern of city developments. The study aims to detect spatial analogies as well as differences for the four largest Mexican urban agglomerations.
\end{abstract}

\section{INTRODUCTION}

Since 2008 and for the first time in history, the world's urban population has outnumbered the rural population. According to the United Nations' World Urbanization Prospects [1] this trend will continue to rise decisively over the next decades. In addition, the number of megacities urban agglomerations with more than 10 million inhabitants is estimated to increase from 21 to 27 by 2025 [2].

Although urbanization is a worldwide phenomenon, it is especially dynamic in developing and threshold countries such as India, Egypt, Brasil or Mexico, where cities have experienced an enormous growth over the last 40 years. Latin America and the Caribbean are the most urbanized cultural region in the developing world, with $77 \%$ of its population living in cities [2]. Thus, Mexico provides a good example for this investigation as it continues to undergo urbanization since the beginning of the $20^{\text {th }}$ century [3].

Remote sensing techniques have already proven useful for mapping urban areas at various scales and obtaining data for the analysis of urban land cover change [4]. Recent research has used remotely sensed images to quantitatively describe the spatial structure or pattern of urban environments and characterise urban morphology. Spatial metrics prove efficient in the description, analysis, and modelling of urban form and its changes [5]. Zonal statistics as well as gradient analysis extend the capabilities to measure spatial configurations [6].
In this study we specifically address the following research questions:

(1) How can spatiotemporal urban growth be quantified using remote sensing?

(2) Does spatial urbanization correspond to the demographic development of a city?

(3) How can spatial urban patterns and their development be quantified over time?

(4) Is there a spatiotemporal analogy for cities within the same cultural region?

\section{StUdy AREA AND DATA SETS}

Measured by population, the four largest cities of Mexico, namely Mexico City, Guadalajara, Monterrey and Puebla de Zaragoza are used as study sites. Due to it's hegemony as the economic, political and social center of the country, Mexico City plays an outstanding role in the national urban system. With over 21.1 million inhabitants, Mexico City (Ciudad de Mexico) was ranked the third most populous city of the world in 2009 and belongs to the most densely populated regions in Mexico. The recent development of Mexico City has been characterised by slower demographic growth than projected. In the year 2000, the registered 18 million inhabitants fell short of the projected 25-27 million [1].

Just as in Mexico City, immense population growth took place in Guadalajara, Monterrey and Puebla, starting from relatively equal numbers ranging from 200.000 to 400.000 inhabitants in 1950. The metropolitan area of Guadalajara (4.4 million inhabitants) developed the fastest until today, followed by Monterrey (3.9 million) and Puebla (2.3 million). The demographic developments of the metropolitan areas of Guadalajara, Monterrey and Puebla are relatively similar, rising constantly over the last 60 years with population numbers increasing roughly tenfold until 2005 [1].

In this study, spatio-temporal urbanization was monitored using imagery from the optical Landsat series of sensors (the Multispectral Scanner (MSS), the Thematic Mapper (TM) and the Enhanced Thematic Mapper (ETM+)) as well as the stripmap acquisition mode data of the German radar sensor TerraSAR-X. The geometric capabilities of Landsat and TerraSAR-X data are not cluttered with microscopic detail, but let us differentiate urbanised and non-urbanised areas with high accuracy. With it monitoring of spatial urban 
development since the 1970 s until today with time intervals of circa 10 years is possible.

\section{METHODOLOGY}

The methodology is subdivided in two steps: 1) Classification of the multi-temporal remote sensing data and change detection 2) Spatial pattern analysis.

1) An object-oriented, hierarchical classification approach extracting the classes 'built-up areas', 'non built-up areas' and 'water' was performed separately on the Landsat images [6]. The approach utilises spectral, shape, texture and context features. The specific classification of optical Landsat TM and ETM+ data was executed using an eCognition Architect solution. This graphical user interface (GUI) replaces fixed with variable decision tree rules and has proved to be applicable on different test sites like Mexico City, Istanbul and Cairo [7]. To enrich the information value for classification of Landsat MSS data, principal component analysis and Tasseled Cap Transformation were calculated to improve classification accuracy.

A fully-automated pixel-based classification algorithm was applied to the Terra-SAR-X data to delineate urbanised from non-urbanised areas [8]. The urban footprint is extracted by analysing the speckle characteristics of a TerraSAR-X scene, based on an estimation of the local coefficient of variation and the fading texture of the whole scene. The basic concept is to extract reliable urban features for the urban footprint classification such as seed points, which are represented by bright point scatterers (corner reflectors). The 'urban seeds' particularly depict the reflection information of vertical structures. Urban seeds are extracted from intensity and texture, derived from a two-tiered speckle divergence calculation with a 9x9 and a $35 \times 35$ window [8]. To derive the urban footprint, intensity, context and texture information are combined to densify and generalize the detected urban seeds.

For urban change detection, post classification comparison was found to be the most accurate procedure as it has the advantage of indicating the nature of changes [9]. Post classification comparison of urban footprints involves the detection of differences between two independent classification results. Subsequent to multitemporal classifications, a comparison of the categorizations was performed [10]. The change detection (i. e. Fig. 1) serves as basic geo-information layer for a spatio-temporal and crosscity analysis of the urban footprints.

2) For the spatial pattern analysis we developed a four-step analysis based on absolute growth analysis, zonal statistics, gradient analysis and landscape metrics.

First, absolute spatial urban growth is derived from the change detection and related to population statistics over time. Thus, relationships between both parameters aim at the detection of similar or differing trends. Within available time steps from remote sensing results linear interpolation is applied.

Second, zonal statistics provide a possibility to derive spatial parameters and measures with respect to location. The built-up density is a measure to characterise spatial urban pattern and structure. Densities vary substantially from city to city and from urban centers to peripheral areas [11]. A ringbuffer analysis employs six artificial concentric rings with $5 \mathrm{~km}$-intervals around the center creating comparable zones from the urban core to the fringes. The center of each particular city is defined as the respective Zocalo, which is the central plaza of a typical Mexican city [12]. Built-up density is calculated as ratio between the areas of the particular ring with water areas omitted and the urbanized areas.

Third, gradient analysis aims to identify mono- or polycentric spatial growth types. Therefore, from the classification results, a scan algorithm counts the number of urbanized pixels per row and per column in comparison to non-urbanized areas. The result of the scan, calculated in percent, is displayed as a continuous graph over the particular spatial location in $\mathrm{x}$ and $\mathrm{y}$ direction [13]. With respect to location - the urban center (Zocalo) exactly determines the corresponding positions in $\mathrm{x}$ and $\mathrm{y}$ direction - the two plots in $\mathrm{x}$ and $\mathrm{y}$ directions are added to integrate the spatial information of urbanized gradients into one diagram. Peaks indicate a spatial urban center or sub-center.

Fourth, landscape metrics (or spatial metrics) measure and describe the spatial structure of patches, classes of patches or entire patch mosaics [14]. Although these indices of landscape pattern have been used for decades in ecology, only recently they have been applied specifically for the study of urban morphology [15]. Landscape metrics aim at learning the spatial mechanisms and complex processes of urban growth by finding analogies and differences in the historical development of cities under consideration [11].

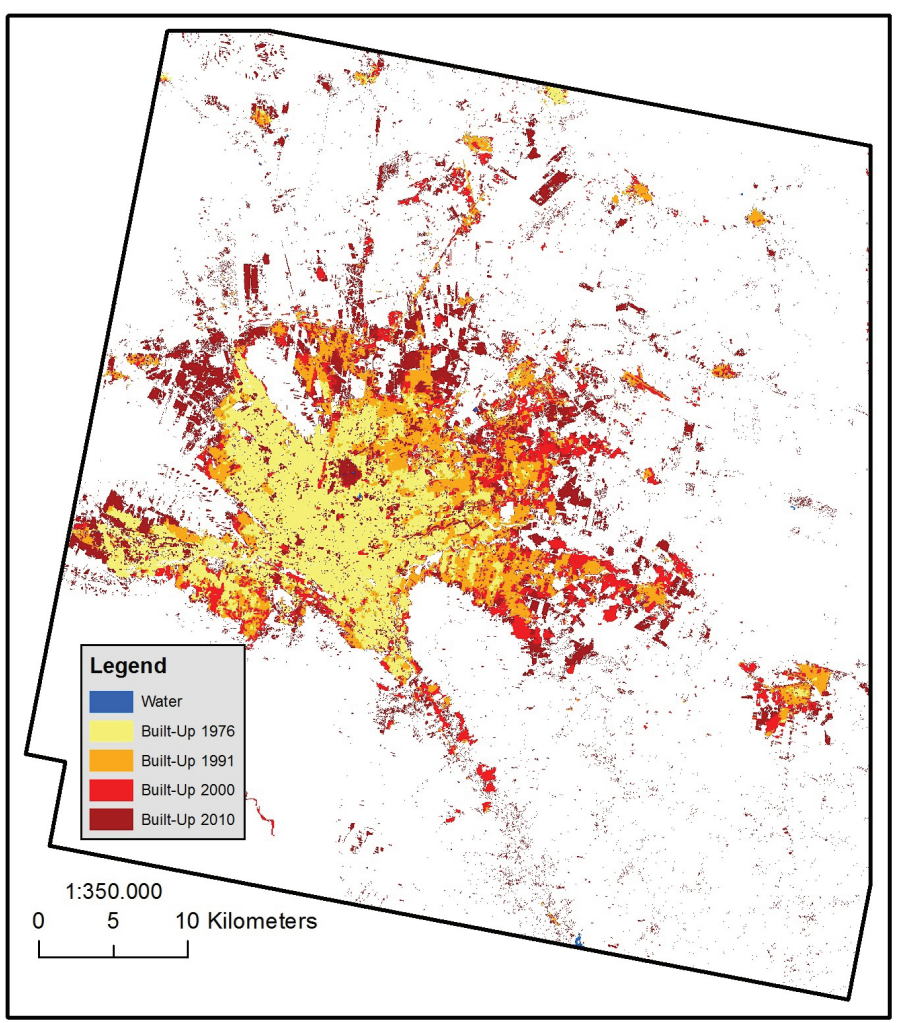

Figure 1. Change detection displaying spatial urbanization in Monterrey 
In this study, we apply the Largest Patch Index (LPI) and the Landscape Shape Index (LSI). The LPI belongs to the group of "area metrics", which quantify landscape composition $[15,16]$. The LPI equals the percentage of the landscape comprised by the largest patch [17]. The LPI was selected to measure the dominance of the largest patch. It reaches $100 \%$, if the entire landscape consists of a single patch and approaches zero, as the largest patch becomes smaller. In addition to the LPI, the LSI was chosen which belongs to the group of "shape metrics" and is a measure of landscape configuration by complexity of patch shape. This index measures the perimeter-to-area ratio for the landscape as a whole. The LSI equals the total length of edge (or perimeter) of a class, given in number of cell surfaces, divided by the minimum length of class edge possible for a maximally aggregated class, which is achieved when a class is maximally clumped into a single, compact square patch. The LSI reaches 1 if the urbanised area comprises one single compact square area. If the landscape consists of disperse patches of complex shape the LSI will be large. Thus, the LSI is a measure of clumpiness or complexity of urban growth [17].

\section{RESUlTS, VALIDATION AND DISCUSSION}

The presented methods are applied to the four major urban agglomerations in Mexico. A sample result of the multitemporal change detection is displayed by figure 1 showing urban sprawl as well as re-densification processes in the urban core of Monterrey from 1976 until 2010. The city is extensively sprawling, displaying finger-like growth axis and a small satellite town to the east. But, in general Monterrey has a rather compact urban footprint.

The small-scale spatial variety of land surfaces, coarse geometric resolution and mixed spectral information in certain pixels of the Landsat imageries is limiting the accuracy of the classification result. The various effects in SAR imagery introduce further uncertainties for classification. As ground truth data was not available for this study, the accuracies of the classification results were assessed by a randomization of 300 checkpoints per class per city and a subsequent visual verification process. Table 1 shows the results of the overall accuracy assessment for the various classification results.

\begin{tabular}{|l||l||c||c||c||}
\hline $\begin{array}{l}\text { Overall } \\
\text { Accuracy }\end{array}$ & $\begin{array}{l}\text { Landsat } \\
\text { MSS }\end{array}$ & $\begin{array}{l}\text { Landsat } \\
\text { TM }\end{array}$ & $\begin{array}{l}\text { Landsat } \\
\text { ETM+ }\end{array}$ & TerraSAR-X \\
\hline \hline Mexico City & $88.0 \%$ & $96.0 \%$ & $94.0 \%$ & $97.5 \%$ \\
\hline \hline Guadalajara & $95.0 \%$ & $96.0 \%$ & $93.7 \%$ & $97.0 \%$ \\
\hline \hline Monterrey & $95.0 \%$ & $97.7 \%$ & $85.7 \%$ & $96.0 \%$ \\
\hline \hline Puebla & $97.0 \%$ & $95.0 \%$ & $95.7 \%$ & $94.0 \%$ \\
\hline
\end{tabular}

Table 1. Accuracy assessment of the urban footprint classifications

The kappa coefficient also shows strong agreement between classification results and the reference information with varying values from 0.64 to 0.92 . The urban footprint classifications have to be understood as a coarse approximation of urbanized areas and not as an exact classification of a building mask. Still, the requirements of mapping the large city footprint, its spatial dimension and the spatial developments over the years are fulfilled. The multisensoral earth observation data provide sufficient information for a correct detection of urban change and its spatial configuration.

The first and most obvious analysis is derived from the change detection, comparing the spatial growth of the four Mexican cities over the past 35 years. The plot against population development over time reveals an increase of spatial urbanization compared to population growth, indicating an increasing need of space per person (Fig. 2).

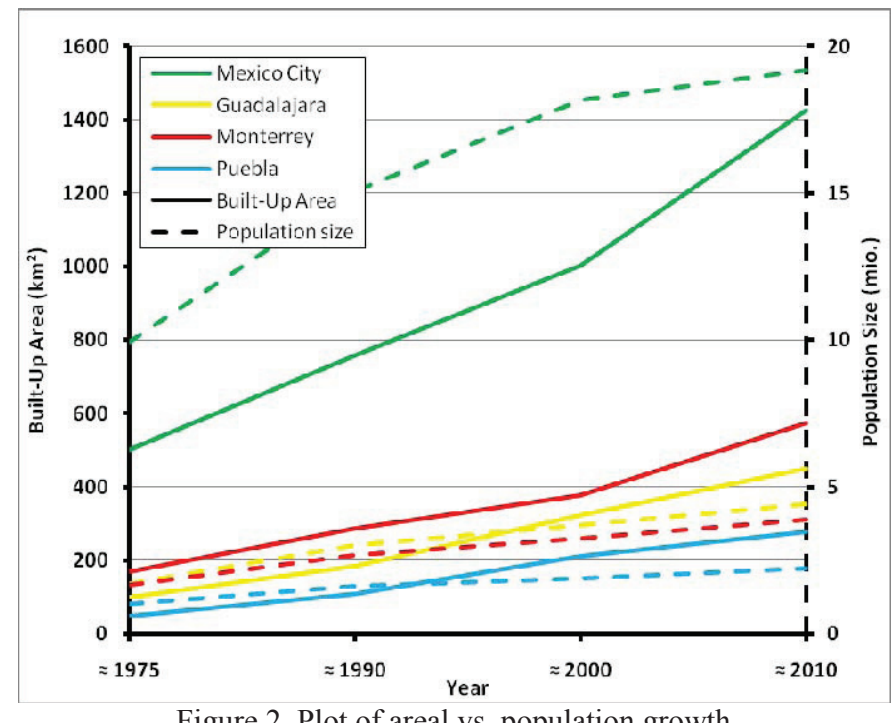

Figure 2. Plot of areal vs. population growth

For location-based analysis, zonal statistics are calculated and compared (Fig. 3). In general, a decreasing built-up density with increasing distance to the urban center is detected for all four cities. Monterrey, Guadalajara and Puebla show a similar abrupt decrease in density around the central zone and low built-up densities in the outer rings. In comparison, megacity Mexico City clearly reveals its large urban core by a slow decrease of density with rising distance to the center and built-up densities higher than $55 \%$ even in the $6^{\text {th }}$ ring. It also becomes obvious that spatial growth rates are highest in distant rings of the mega city, while for the three smaller cities, absolute spatial growth is still highest in or close to the urban core.
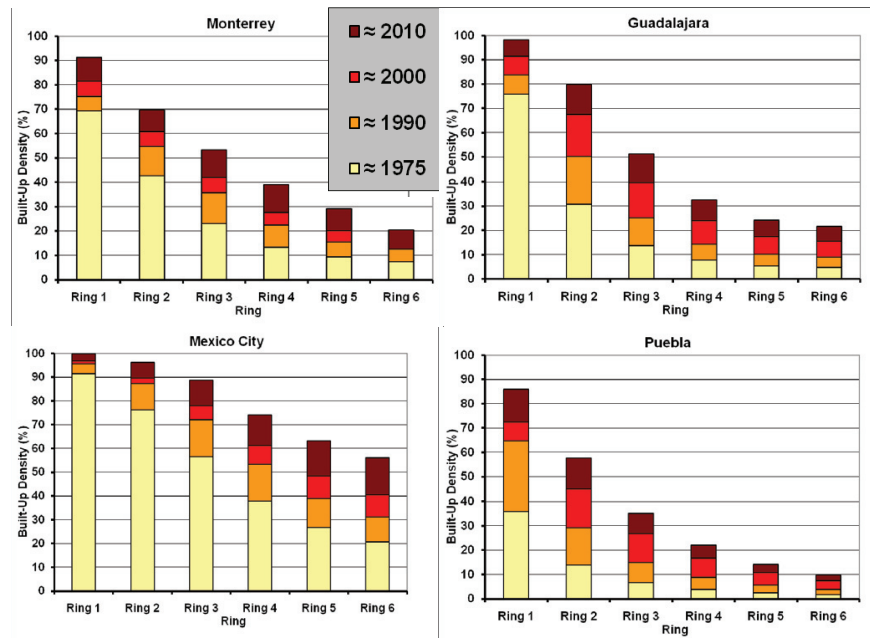

Figure 3. Zonal statistics using ring buffers 
Location-based gradient analysis aims at spatially detecting urban centers or sub-centers and their development over time (peaks in the histogram). Results of the histogram analysis clearly reveal a polycentric structure for Monterrey and Mexico City with a dominant urban core for both cities. Their polycentric structure only developed recently, while all cities showed to a large extent a monocentric urban pattern in the 1970s. However, also Guadalajara and Puebla display trends to a polycentric urban configuration in the latest time step.
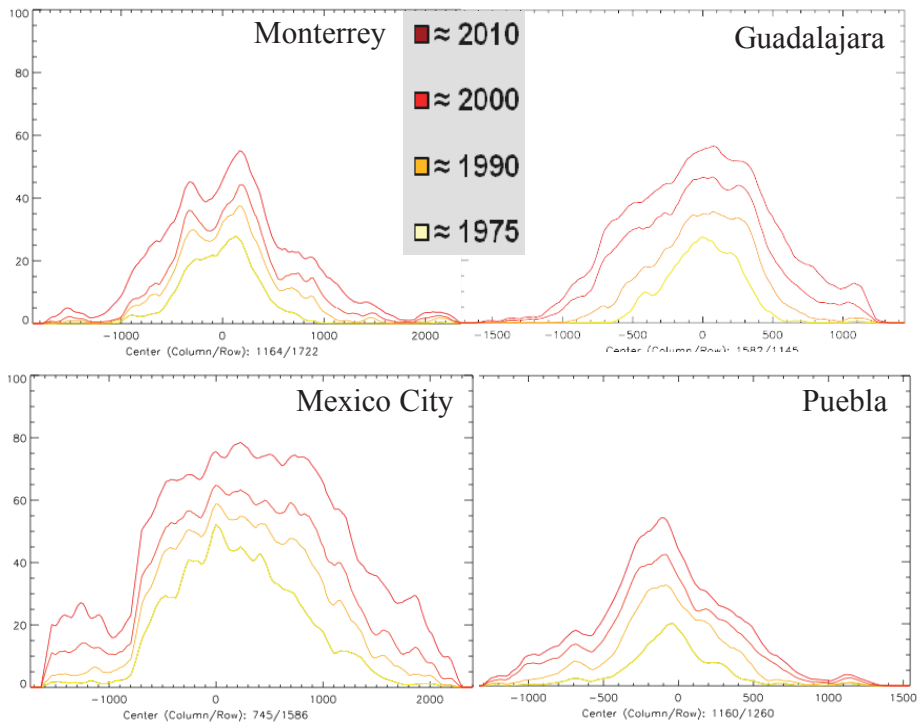

Figure 4. Gradient analysis

Finally, landscape metrics are displayed to quantify spatial urban configuration. The LPI reveals a rising dominance of the main urban patch for all cities. It is especially high in Mexico City, where the limited space within the cordillera enforces redensification processes and thus a coalescence of the urban core. The sprawling three smaller cities also display a rising complexity in spatial configuration, while the redensification in the megacity recently shows a decrease in complexity.

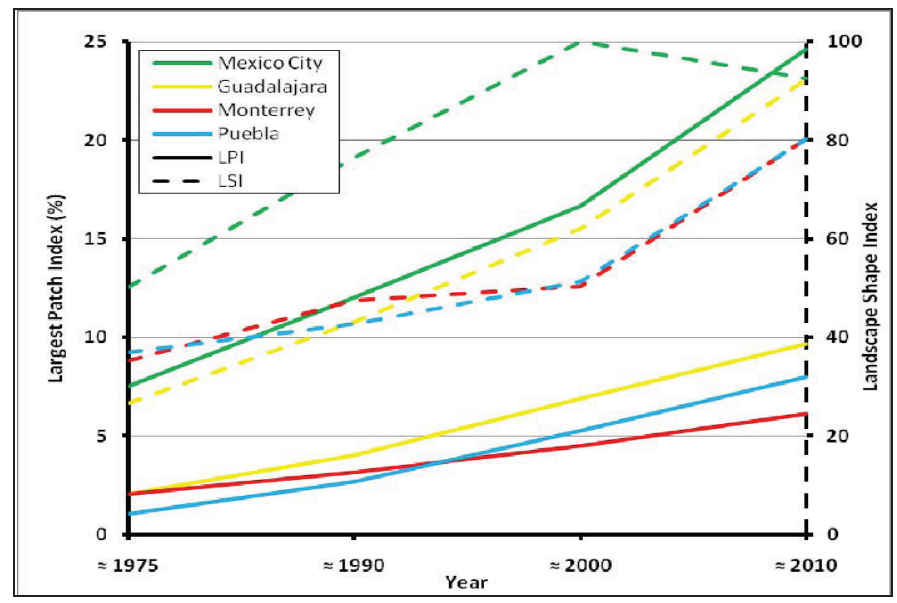

Figure 4. Landscape metrics

The results from the various methodologies clearly disclose analogies for the cities of Monterrey, Guadalajara and Puebla. The cities show comparable increase in population, spatial growth and a tendency to higher urbanization rates per person. Furthermore spatial configuration from the urban core to the periphery shows similar gradients in built-up density or a tendency from mono- to polycentric spatial configuration. Mexico City clearly differs due to its dimension; its main growth rates in peripheral areas as well as its polycentric configuration.

\section{CONCLUSION}

The study has demonstrated that urbanization and its spatiotemporal dimension and structure can be quantified and compared across cities using a combination of absolute parameters, zonal statistics, gradient analysis and landscape metrics. Landsat as well as TerraSAR-X data proved to be an independent, area-wide, long-time, up-to-date and (with respect to the limited geometric resolution) adequate data source for the analysis of large and fast-changing areas of Mexican cities. Comparative studies are crucial to detect urban growth trajectories across cities and to learn for sustainable urban planning from more than one individual city.

\section{REFERENCES}

[1] United Nations (2007): World urbanizationprospects, the 2007 revision. New York.

[2] United Nations Human Settlemet Programme (2008): State of the world's cities 2008/2009 - Harmonious cities. Nairobi.

[3] Champion, T., Hugo, G. (2004): New forms of urbanization - Beyond the urban-rural dichotomy. Aldershot: Ashgate.

[4] Donnay, J. P., Barnsley, M. J., \& Longley, P. A. (2001). Remote sensing and urban analysis. London: Taylor and Francis.

[5] Herold, M., Goldstein, N. C., \& Clarke, K. C. (2003): The spatiotemporal form of urban growth: Measurement, analysis and modelling. Remote Sensing of Environment, 86, 286-302.

[6] Taubenböck, H. (2008): Vulnerabilitätsabschätzung der Megacity Istanbul mit Methoden der Fernerkundung. Dissertation. Universität Würzburg; p. 178. ISBN-10: 3639083180.

[7] Abelen, S., Taubenböck, H. \& Stilla, U. (2011): Multi-scene urban area classification via decision tree adjustment. Joint Urban Remote Sensing Event 2011 (to appear in the same proceedings).. JURSE Conference Munich.

[8] Esch, T., Thiel, M., Schenk, A., Roth, A., Müller, A., Dech, S. (2010): Delineation of urban footprints from TerraSAR-X data by analyzing speckle characteristics and intensity information. In: IEEE Transactions on Geoscience and Remote Sensing, Vol. 48, Issue 2, p. 905-916.

[9] Mas, J.F. (1999): Monitoring landcover changes: A comparison of change detection techniques. In: International Journal of remote sensing, Vol. 20, p. 139-152.

[10] Lunetta, R.S., Elvidge, C.D. (1998): Remote sensing change detection environmental monitoring methods and applications. Chelsea.

[11] Taubenböck, H., Wegmann, M., Roth, A., Mehl, H. \& Dech, S. (2009): Urbanization in India - Spatiotemporal analysis using remote sensing data. Computers, Environment and Urban Systems 33. pp 179-188.

[12] Pacione, M. (2005): Urban Geography. New York

[13] Taubenböck, H., Esch, T, Wurm, M., Thiel, M., Ullmann, T., Roth, A., Schmidt, M., Mehl H. \& Dech, S. (2008): Urban structure analysis of mega city Mexico City using multi-sensoral remote sensing data. In: Proceedings of SPIE-Europe Conference, Cardiff, Wales, p. 8-20.

[14] Leitaio, A.B., Miller, J., Ahern, J., McCarigal, K. (2006): Measuring landscape: a planner's handbook. Washington.

[15] Netzband, M., Stefanov, W.L., Redman, C. (2007): Applied remote sensing for urban planning, governance and sustainability. Heidelberg.

[16] McGarigal, K., Marks, B. (1995): FRAGSTATS: spatial pattern analysis programm for quantifying landscape structure. Portland.

[17] McGarigal K., Cushman, S.A., Neel, M.C. and Ene E. (2002): FRAGSTATS: spatial pattern analysis programm for categorical maps. Computer software; University of Massachusetts, Amherst. 